Umfrage zum Patientenrechtegesetz

\title{
Jeder dritte Arzt ist skeptisch
}

\author{
In Sachen Patientenrechtegesetz herrscht noch \\ große Unsicherheit in den Praxen. Wie eine aktu- \\ elle Umfrage bei mehr als 500 Ärzten zeigt, fühlen \\ sich nur wenige Ärzte gut über das Gesetz infor- \\ miert. Mehr als jeder dritte Arzt fürchtet sogar \\ negative Auswirkungen auf seine Praxis.
}

R auf und runter diskutiert wurde das Patientenrechtegesetz im vergangenen Jahr. Paragrafenweise hatten es Politiker, Patienten- und Ärztevertreter immer wieder auseinandergenommen und nachjustiert bis es dann in diesem Frühjahr tatsächlich in Kraft treten konnte. Trotzdem fühlen sich die Ärzte alles andere als sicher in dem neuen Gesetz.

Drei Viertel der teilnehmenden Ärzte kennen die Inhalte nur grob oder fühlen sich sogar schlecht informiert, so das Ergebnis der gemeinsamen Leserumfrage von „Ärzte Zeitung" und CompuGroup Medical zum Patientenrechtegesetz. Dabei haben sich an der nicht repräsentativen Studie immerhin 551 Ärzte beteiligt - das zeigt, dass das Thema Patientenrechtegesetz in den Praxen doch eine hohe Relevanz hat. Rund 40 \% können die Auswirkungen, die das Gesetz auf den Praxisalltag künftig haben wird, zwar noch nicht abschätzen. $38 \%$ gehen aber davon aus, dass es negative Folgen für die Praxen haben wird.

Jeder zweite der teilnehmenden Ärzte war dabei schon einmal mit Streitfällen konfrontiert, bei denen er als Arzt nachweisen musste, ordnungsgemäß gearbeitet zu haben. Das neue Gesetz könnte diese Situation - so die Meinung der Mehrzahl der Umfrageteilnehmer - noch verschärfen. Denn rund $62 \%$ gehen davon aus, dass Mediziner die Beweislastumkehr in Verfahren um mögliche Behandlungsfehler künftig schneller trifft. Ärzte konnten zwar auch schon vor dem Inkrafttreten des Gesetzes in die Situation kommen, dass sie in volle Beweispflicht rutschten. Doch das hing stark vom jeweiligen Gericht ab.

Was hier hilft, ist eine lückenlose Dokumentation. Doch genau hier hapert es in den Praxen scheinbar noch - oder zumindest herrscht hier eine gewisse Unsicherheit, wie die Umfrage zeigt. Gerade einmal ein Fünftel der Umfrageteilnehmer archiviert die Praxisdaten bereits revisionssicher. Rund 66 Prozent sind sich unsicher, ob ihre Archivierung revisionssicher ist. Dass sich die Software-Anbieter längst mit dem Thema revisionssichere Archivierung und Patientenrechtegesetz auseinandersetzen, zeigt das Beispiel der CompuGroup Medical. Wie das Unternehmen berichtet, gibt es sowohl für die Arzt-, als auch die Klinik- und Zahnarztinformationssysteme bereits passende EDV-Lösungen. 